\title{
Aerodynamic Flow Visualisation Over Surfaces Using Smoke Separation Method from Ansys Fluent
}

\author{
Virendra Talele ${ }^{1}$, Niranjan Sonawane ${ }^{2}$, Omkar Chavan $^{3}$, Akash Divate $^{4}$, Niraj Badhe ${ }^{5}$, Mirza Adil Ansar Beg ${ }^{6}$, Harshal Patil $^{7}$ \\ 1,2,3,4, U.G, B.Tech Student, Department of Mechanical Engineering, MIT School of Engineering, MIT ADT \\ University, Pune, Maharashtra, India.
}

\begin{abstract}
In the present study, three workbench problem for turbulence modeling with high Reynolds number is used to determine the behavior of fluid flow around the surfaces. The cases for simulation is developed using Ansys workbench CFD fluent module. The computational results are obtained using solution sets of high Reynolds number with the LagrangianEulerian (LE) approach of point particle tracking system in Nevers stoke RANS Equation. The effect of flow pattern around the surface and its kinetic behavior of fluid is evaluated in post-process method of results. By observation, it has been tabulated that fluid flow separation is arousal at the corner end of all surfaces which happens due to evoking of a large adverse pressure gradient.
\end{abstract}

Keywords:- Ansys, LE, RANS, Reynolds number, Pressure gradients.

\section{INTRODUCTION}

Investigating behavior and flow separation of fluid and microfluid levels is very important in producing upscale optimize models in Hydraulics, Pneumatics, and Aerodynamic investigations. The mode of Numerical investigation in CFD applications has present more interest in engineering applications due to added several benefits ranging from saving dependence cost of manufacturing prototypes, Solving for different iterative cases. In (2006) HYUNGMIN PARK and et. Have presented a detailed view of flow over the bluff body and the method of formdrag reduction in the flow over a two-dimensional bluff body [1]. In (2020) S.Sadeghipour and his team have presented a research study with a wind tunnel experimental setup to validate a flow around a bluff body cylinder which is having the porous structure in the material, they have validated results for different shapes like square, cylinder [2]. In (2008) Haecheon Choi et al. have presented a review article based on the various method of controlling flow over the bluff body around a cylinder and spiral shapes by 3D forcing, active feedback control with symmetrical jet method [3]. In (2019) Zhongyang Qi and Yankui Wang have presented a study on Control of asymmetric flow over a blunt-nosed slender body where they have used an innovatively designed device and tested in experimental setup for a high varying angle of attack, The result of this study is to determine the vortice characteristics around the surface in forcing asymmetrical way [4]. In (2016) $\mathrm{M}$. Elkhoury has presented a research CFD based study of a detailed investigation of vortices behavior over different sets of the turbulent model equation, in this case, the have used a square shape wall mounted bluff body [5]. In (2017) Nandu Gopan and Meheboob Alam have presented a study with experimental and simulation basis nature and behavior of bluff body going under the shock wave separation under the granular airstream structure for local Mach number ranging from the subsonic and supersonic region [6]. In 2017

Robert Maduta and Suad Jakirlic have presented a study of flow separation around an Ahmed body with having slant edge of $25^{\circ}$ whereas they have validated results with evidence of RANS equation and set of different turbulence wall functions [7]. In (2008) M. Minguez et al. had an aerodynamic computational investigation of flow over Ahmed body to determine the particle separation using the pseudogenization method where they have using LES turbulence modeling appearance for equation [8].

\section{$>$ Problem Statement}

The Present research study is focussed on the flow separation of air fluids from the bluff bodies. In this investigation, we have implemented a turbulence model with near-wall treatment having a set of RANS equations. Of high Reynolds number. The shape like the structure of Cylinder, square, triangle is used in the present simulation study. The cases have been set up using ANSYS fluent R 18.0. This research is intended to benefit Aerodynamic Researchers because by understanding flow separation from the geometry we can optimize and control the creation of vortices in the system.

\section{METHODOLOGY}

The present research paper is focused in validating results by using Commercial CFD Software ANSYS Fluent which is having commercial CFD solver codes and a set of turbulence modeling equations. In the present work Kepsilon model is used for a high range of Reynolds number with having RANS equations near wall treatment method. The setup of the solution and CFD investigation is divided in three aspects a) Surface preparation, b) solution tuning, and c) Post-Processing results with scientific contours.

\section{A. Surface preparation \\ In surface preparation, geometry creation and generation of the grid are the two main aspects. In the present research work, a rectangular grid has been established which consists of tetrahedron mesh spectral mesh of $3 \mathrm{~mm}$ with maintaining skewness below 0.98 . Figure 1 and Figure 2, Shows the grid generation and meshing of different surface.}



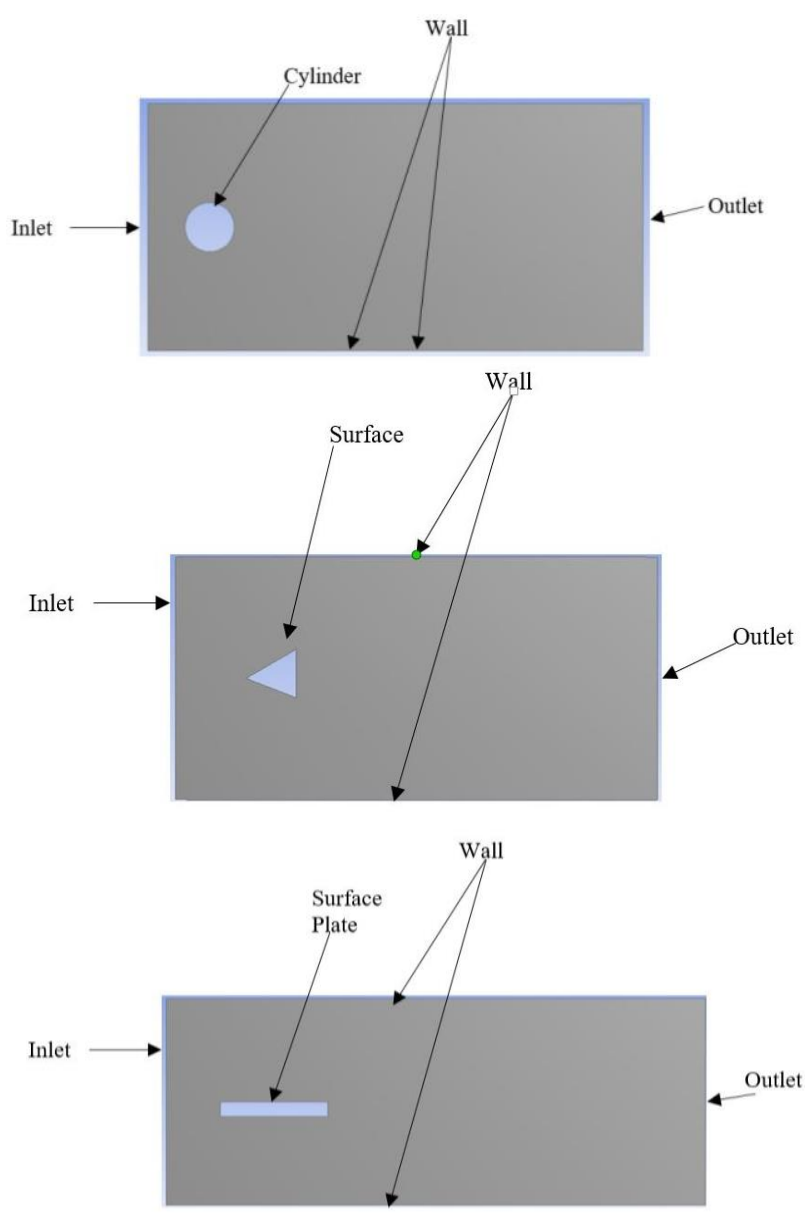

Fig 1:- Surface preparation.
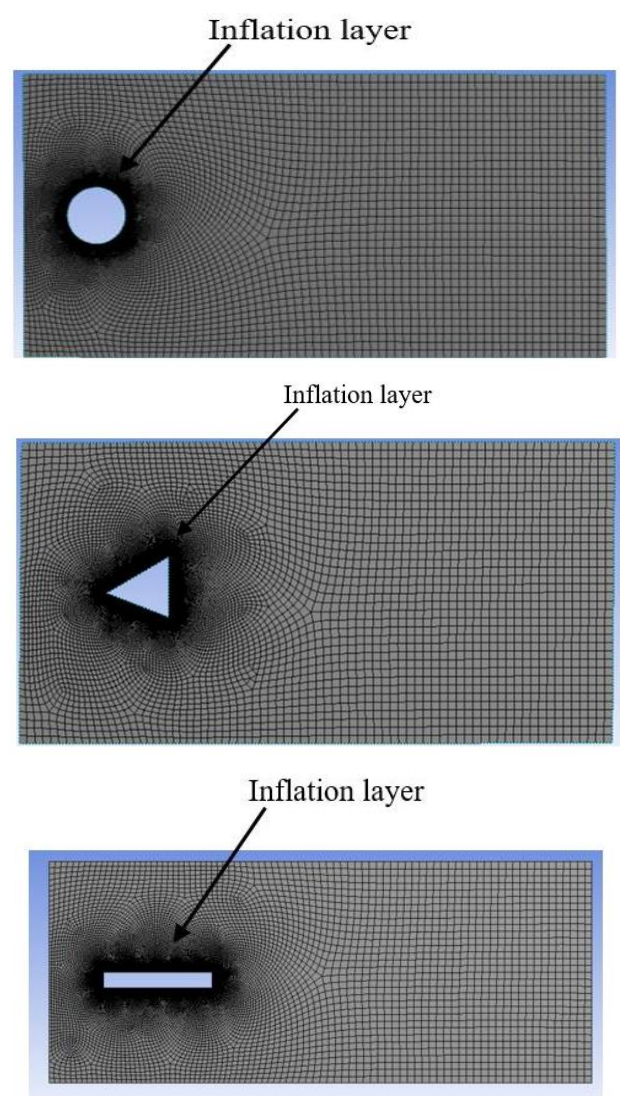

Fig 2:- Grid Generation and meshing

\section{B. Solution Monitoring and Turbulence tunning.}

The solution tuning and monitoring are one of the most important aspects in CFD simulation because it determines the quality of the numerical method that has been set behind the black box. If there is misinformative data in preparing mathematical model during solution preparation it causes the hamper in our post-process result and divergence to occur during the process. To prepare the most optimum converging result the input data from pre-processing and mathematical inputs must be tuned properly.

In the present paper, bluff body is analyzed for the turbulence flow over the surface. In general its hard to predict the intensity of turbulence and the direction of flow over the surface in practical condition but we can predict the replica of turbulence flow over the surface using fine-tune mathematical model based on stokes equation which includes a time-averaged conversation in the equation of transient manner due to time dependant behavior.

\section{Governing Equation -}

$\frac{\partial \bar{u}}{\partial x}+\frac{\partial \bar{v}}{\partial y}=0$

$\rho\left(\frac{\partial \bar{u}}{\partial t}+\bar{u} \frac{\partial \bar{u}}{\partial x}+\bar{v} \frac{\partial \bar{u}}{\partial y}\right)=-\frac{\partial \bar{P}}{\partial x}+\left(\mu+\mu_{t}\right)\left(\frac{\partial^{2} \bar{u}}{\partial x^{2}}+\frac{\partial^{2} \bar{u}}{\partial y^{2}}\right)$

$\rho\left(\frac{\partial \bar{v}}{\partial t}+\bar{u} \frac{\partial \bar{v}}{\partial x}+\bar{v} \frac{\partial \bar{v}}{\partial y}\right)=-\frac{\partial \bar{P}}{\partial y}+\left(\mu+\mu_{t}\right)\left(\frac{\partial^{2} \bar{v}}{\partial x^{2}}+\frac{\partial^{2} \bar{v}}{\partial y^{2}}\right)$

$\frac{\partial \bar{T}}{\partial t}+\bar{u} \frac{\partial \bar{T}}{\partial x}+\bar{v} \frac{\partial \bar{T}}{\partial y}=\left(\alpha+\alpha_{t}\right)\left(\frac{\partial^{2} \bar{T}}{\partial x^{2}}+\frac{\partial^{2} \bar{T}}{\partial y^{2}}\right)$

where $\mathrm{q}$ is density $(\mathrm{kg} / \mathrm{m} 3), \mathrm{x}$ and $\mathrm{y}$ represent the Cartesian spatial coordinates on the $\mathrm{x}$ and $\mathrm{y}$-axis directions (m); $\mathrm{u}$ and $\mathrm{v}$ are the velocity components on the $\mathrm{x}$ and $\mathrm{y}$ axis directions, respectively $(\mathrm{m} / \mathrm{s}), \mathrm{P}$ is the pressure $(\mathrm{N} / \mathrm{m} 2)$, $\mathrm{T}$ is the temperature $(\mathrm{K}), \mathrm{cp} \mathrm{cp}$ is the specific heat at constant pressure $(\mathrm{J} / \mathrm{kg} \mathrm{K})$, a is the thermal difusity of the fluid $(\mathrm{m} 2 / \mathrm{s})$, at is the turbulent thermal difusity $(\mathrm{m} 2 / \mathrm{s}), \mu$ is the dynamic viscosity $(\mathrm{kg} /(\mathrm{m} \mathrm{s})), \mu \mathrm{t}$ is the turbulent dynamic viscosity $(\mathrm{kg} /(\mathrm{m} \mathrm{s}))$ and overbar is the time averaged operator [9].

\section{Turbulence Setup -}

In the present paper, bluff body is analyzed for flow over high Reynolds number ranging from in the range of subsonic to supersonic i.e Mach < 1.0 to Mach > 1.0 The setup used for turbulence modeling is high order derivative second-order upwind partial differential equation.

The original input of the K-Epsilon model was the improvement of the mix length model which states the fine algebraical prescribe turbulent length and intensity of arousal over the flow[10]. 
For turbulent kinetic energy k -

$$
\frac{\partial(\rho k)}{\partial t}+\frac{\partial\left(\rho k u_{i}\right)}{\partial x_{i}}=\frac{\partial}{\partial x_{j}}\left[\frac{\mu_{t}}{\sigma_{k}} \frac{\partial k}{\partial x_{j}}\right]+2 \mu_{t} E_{i j} E_{i j}-\rho \varepsilon
$$

For dissipation E-

$$
\frac{\partial(\rho \varepsilon)}{\partial t}+\frac{\partial\left(\rho \varepsilon u_{i}\right)}{\partial x_{i}}=\frac{\partial}{\partial x_{j}}\left[\frac{\mu_{t}}{\sigma_{\varepsilon}} \frac{\partial \varepsilon}{\partial x_{j}}\right]+C_{1 \varepsilon} \frac{\varepsilon}{k} 2 \mu_{t} E_{i j} E_{i j}-C_{2 \varepsilon} \rho \frac{\varepsilon^{2}}{k}
$$

\section{Post-Processing}

Post-Processing is a very crucial step in Computational modeling because setting up an efficient background for the solutions it helps to optimize and validate objective meeting results in a proper manner.

In the present investigation, the aim is to find out the separation of viscous flow over the shapes. Through the flow separation microscopic analysis in the flow pattern, it has been observed that intensity and duration of flow separation occur in different manner for different shapes of the bluff body. The results has been validated through the post prcess solver contour for Eddy Viscosity and Kinetic behavior of fluid flowing over the region.

The following results show the flow separation over the surface in transient modeling and formation vortices due to von-karman effect in the flow models.

\section{RESULTS}

The scientific evidence of flow separation over the edges is shown in the result which is active in postprocessing of the solution.

Contour 1) - It shows the flow separation over the cylinder for the high value of Reynolds number under an unsteady flow of transient timestep condition. It ha been observed the von-karma effect is getting increased by increasing the value of Reynolds number. Figure 3 shows the post-process flow separation result of Cylinder.

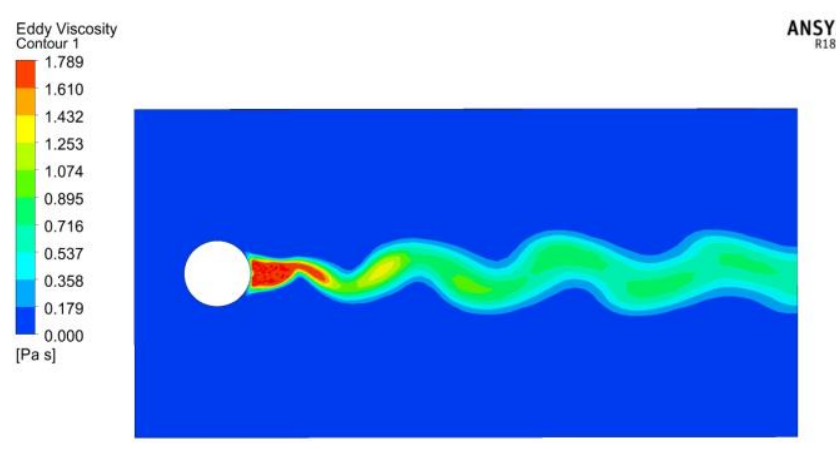

Fig 3:- Flow separation over Cylinder

Contour 2) - It shows the flow separation over the Triangular surface. It has been observed that in triangular shape the flow separation has occurred over the edge of the surface since there is an adverse effect of the pressure gradient is occurring in the wake region which causes the flow separation over the edge in counterfeiting vortex effect.
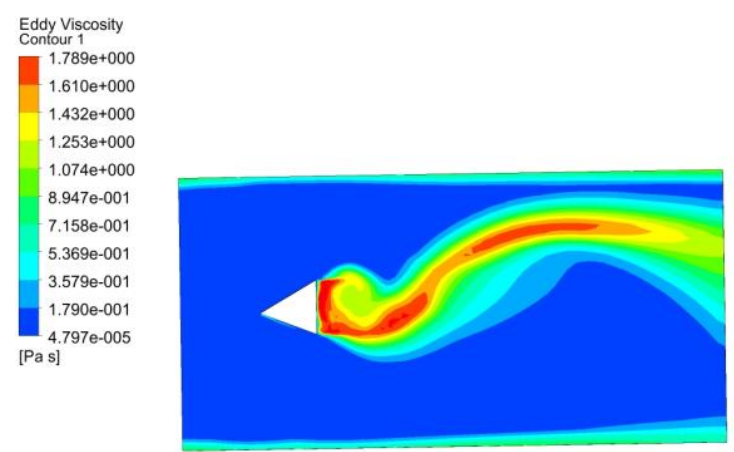

Fig 4:- Flow separation over the triangular surface

Contour 3) - It shows the flow separation over the flat surface body. It has been observed that in case of flat surface there is a delay of flow separation occurs due to viscous flow of conada effect at the leading edge.
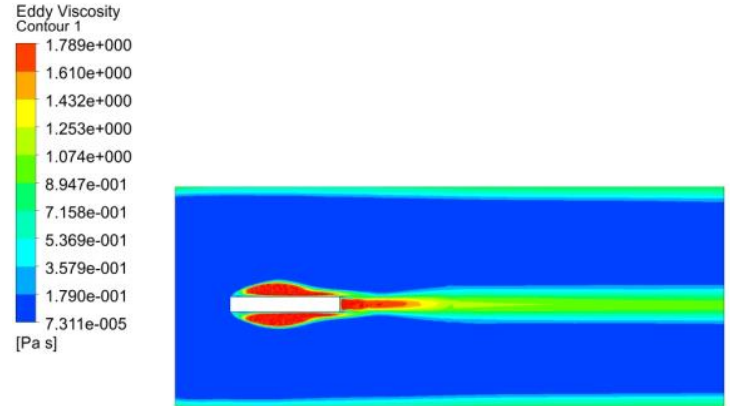

ANSYS

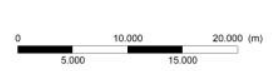

Fig 5:- Shows the flow separation over the flat surface.

\section{CONCLUSION}

Aerodynamic Investigation was carried out to trigger the behavior of flow separation of Air over the different Bluff body shapes, Because the Numerical and flow visualization study of flow separation is very important in engineering problem since it helps out to optimize and validate design according to the constraint limits. From performing this investigation it has been observed that characteristics of flow separation is increased as per increasing Reynolds number from subsonic to supersonic speeds. In case of bluff body the flow separation is occurred in the wake of vortices region, In the case of flow over the triangular surface, it has been observed that flow separation is occurring at the edge of the surface which is due to adverse pressure gradient effect over the body. In the case of Flat plate, it has been observed that there is more delay is flow separation due to the Viscous flow of Coanda effect following over the boundary layer until Reynolds number get increases. 
This presented study is aims to help engineers to solve and optimize the best iterative solution because if the can control the flow separation phenomenon in design it inversely increase the smooth working life span of product.

\section{REFERENCES}

[1]. Hyungmin park $^{1}$, dongkon lee ${ }^{1}$, Woo-pyung jeon ${ }^{2}$, seonghyeon hahn ${ }^{2}$, Jeonglae kim $^{1}$, jungwoo $\mathrm{kim}^{2}$, jin choi $^{1}$ and haecheon choi "Drag reduction in flow over a two-dimensional bluff body with a blunt trailing edge using a new passive device" J. Fluid Mech. (2006), vol. 563, pp. 389-414.

[2]. S. Sadeghipour, S. Alihan Showkat Ali, X. Liu, M. azarpeyvaned, G.R. Thorpe, Control of flows around bluff bodies mediated by porous materials, Experimental Thermal and Fluid Science (2020), doi: https://doi.org/10.1016/j.expthermflusci.2020.110048

[3]. Haecheon Choi ${ }^{1,2}$ Woo-Pyung Jeon, ${ }^{1}$ and Jinsung Kim1 "Control of Flow Over a Bluff Body" Annu. Rev. Fluid Mech. 2008.40:113-139. Pg. 114-139

[4]. Phys. Fluids 31, $037108 \quad$ (2019); https://doi.org/10.1063/1.5080756

[5]. 5. M. Elkhoury “ Assessment of turbulence models for the simulation of turbulent flows past bluff bodies" Journal of Wind Engineering and Industrial Aerodynamics Pg. 10-20

[6]. Nandu Gopan1, and Meheboob Alam1 "Oblique shock waves in granular flows over bluff bodies" EPJ Web of Conferences 140, 03053 (2017)

[7]. Maduta, R. and Jakirlic, S., "Improved RANS Computations of Flow over the $25^{\circ}$-Slant-Angle Ahmed Body," SAE Int. J. Passeng. Cars - Mech. Syst. 10(2):2017, doi:10.4271/2017-01-1523.

[8]. Physics of Fluids (1994-present) 20, 095101 (2008); doi: $10.1063 / 1.2952595$

[9]. F.B. Teixeira a, G. Lorenzini b, , M.R. Errera c, L.A.O. Rocha d, L.A. Isoldi a, E.D. dos Santos a "Constructal Design of triangular arrangements of square bluff bodies under forced convective turbulent flows" International Journal of Heat and Mass Transfer, Pg 521-535. 126 (2018)

[10]. P. Parnaudeau, J. Carlier, D. Heitz, E. Lamballais, Experimental and numerical studies of the flow over a circular cylinder at Reynolds number 3900, Phys.

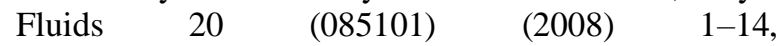
https://doi.org/10.1063/1.2957018.

[11]. D.C. Wilcox, Turbulence Modeling for CFD, third ed., DCW Industries, 2006.

[12]. E. Lamballais, J.H. Silvestrini, Direct numerical simulation of interactions between a mixing layer and a wake around a cylinder, J. Turbul. 3 (2002) 028, https://doi.org/10.1088/1468-5248/3/1/028.

[13]. J. Smagorinsky, General circulation experiments with the primitive equations, Monthly Weather Rev. 91 (1963) 99-164.

[14]. Y. Amarouchene, J.F. Boudet, H. Kellay, Phys. Rev. Lett. 86, 4286 (2001)

[15]. C. Kloss, C. Goniva, A. Hager, S. Amberger, S. Pirker, Prog. Comput. Fluid Dyn. 12, 140 (2012) 